\title{
ILCEA
}

Revue de l'Institut des langues et cultures

d'Europe, Amérique, Afrique, Asie et Australie

$24 \mid 2015$

Lire et écrire ensemble

\section{Des fragments au roman. Une vie de Pierre Ménard}

From Fragments to Novel. Une vie de Pierre Ménard

De los fragmentos a la novela. Una vida de Pierre Menard

\section{Milagros Ezquerro}

\section{(2) OpenEdition}

\section{Journals}

Édition électronique

URL : http://journals.openedition.org/ilcea/3534

DOI : 10.4000/ilcea.3534

ISSN : 2101-0609

Éditeur

UGA Éditions/Université Grenoble Alpes

Édition imprimée

ISBN : 978-2-84310-313-1

ISSN : 1639-6073

Référence électronique

Milagros Ezquerro, «Des fragments au roman. Une vie de Pierre Ménard », ILCEA [En ligne], 24 | 2015, mis en ligne le 03 novembre 2015, consulté le 19 avril 2019. URL : http://journals.openedition.org/ ilcea/3534; DOI : 10.4000/ilcea.3534

Ce document a été généré automatiquement le 19 avril 2019

(c) ILCEA 


\title{
Des fragments au roman. Une vie de Pierre Ménard
}

\author{
From Fragments to Novel. Une vie de Pierre Ménard \\ De los fragmentos a la novela. Una vida de Pierre Menard
}

Milagros Ezquerro

1 Michel Lafon était reconnu, bien avant la publication de l'ouvrage tiré de sa thèse de doctorat d'État, Borges ou la réécriture, comme l'un des meilleurs spécialistes de l'œuvre de Jorge Luis Borges, sur laquelle il a continué à travailler et à fantasmer jusqu'à son dernier souffle. Cette passion fascinée, précoce et obstinée, pour une œuvre et pour un écrivain devenu figure mythique bien avant sa mort, s'exprime dès l'ouverture de l'ouvrage :

Ce siècle est borgésien. On glose l'œuvre de Borges, bien sûr, comme on glose celle de Proust, de Kafka ou de Joyce. Mais surtout on la réécrit. Évidente, l'adaptation: tel texte est transformé en scénario de cinéma, tel autre en dialogue de théâtre, tel autre en livret d'opéra. Tribut somme toute logique payé à la gloire du polygraphe. Plus foisonnante encore et plus complexe, la citation : celle de l'œuvre, mais aussi celle de l'auteur. Car « citer Borges ", ce n'est pas seulement servir tel aphorisme, telle parabole ou tel paradoxe ; ce peut être aussi faire passer sur la page, exhibée ou masquée, la " personne même » qui les proféra. Exemple rare d'un écrivain à ce point confondu avec son œuvre qu'il suffit d'évoquer sa figure, d'écrire son nom ou de projeter son ombre pour qu'aussitôt surgissent les mirages fantastiques qui hantent ses livres. (1990:9)

2 Ce considérable travail, construit autour de la notion, complexe et ondoyante, de « réécriture », n'épuise pas, loin s'en faut, l'addiction borgésienne de Michel Lafon. Non seulement il continue de produire de nombreux essais qui viennent grossir l'abondante bibliographie critique, mais en outre il travaille, en secret, autour de l'une des figures emblématiques du panthéon borgésien, Pierre Ménard. Dix-huit ans après la publication de l'essai Borges ou la réécriture (1990), parait le roman Une vie de Pierre Ménard (2008). C'est le premier roman de Michel Lafon, ce sera aussi, hélas, le dernier. C'est, on peut l'affirmer, l'aboutissement esthétique et intellectuel de sa longue et fructueuse méditation sur l'œuvre de Borges, mais aussi sur lui-même, sur sa vie et son œuvre. On peut dire maintenant que c'est aussi le testament littéraire de Michel Lafon. 
3 Nul n'ignore que tout critique littéraire rêve de passer de l'autre côté du miroir des mots et d'être reconnu comme écrivain à part entière. Beaucoup se contentent de rêver et d'écrire, d'autres rêvent, écrivent et publient poèmes, nouvelles ou romans. C'est ce qu'a fait Michel Lafon : après avoir beaucoup rêvé, beaucoup écrit, il publie un roman, avec un Post-scriptum où l'on peut lire : «Le genre romanesque est décidément, comme auraient dit Ménard, Borges ou tel de leurs amis inoubliables, une facilité - et presque une faiblesse...». Passer de l'essai au roman est donc "une facilité - et presque une faiblesse ", alors pourquoi l'avoir fait ? Le sujet du roman, explicité par le titre, se serait pourtant prêté à un essai biographique et littéraire. Mais ce sujet est loin d'être anodin, surtout quand celui qui l'a choisi est un des meilleurs connaisseurs de l'œuvre de Borges.

\section{De l'essai au roman : le choix du sujet}

C'est dans l'avant-propos qu'est expliqué le sujet des pages qui vont suivre:

Vingt ans tout juste après la disparition de Pierre Ménard, vingt ans sans un jour où je n'aie caressé ce projet - cette mission -, je propose un livre de "témoignage " (le mot me semble bien dérisoire) sur l'ami et sur l'écrivain. Livre bâti un peu à la diable, ce dont j'espère que l'on ne me tiendra pas rigueur : morceaux de sa vie [...], notes prises par moi au fil de nos rencontres et de nos causeries, extraits de correspondances, inédits (de lui ou d'autres), jusqu'à certains documents plus mystérieux que le temps enfui m'autorise sans doute à dévoiler... (2008:14-15)

5 On l'aura compris, l'auteur de ce "témoignage" réfute le caractère fictif de Pierre Ménard, que la nouvelle de Borges «Pierre Menard, autor del Quijote» donne d'abord comme un obscur écrivain nîmois ayant existé, dont il écrit une nécrologie, pour bientôt reconnaître qu'il s'agit d'une fiction totalement inventée. Mais de quel auteur parlonsnous? L'avant-propos est signé à la fin: Maurice Legrand, Montpellier, 24 août 1957. Le signataire se présente comme un disciple de Pierre Ménard, dont le but est de rendre à son maître bien-aimé le statut de personne réelle, statut que Borges lui a donné puis enlevé, en écrivant, en 1939 dans la revue Sur, sa " nécrologie ", dont il a ensuite précisé que c'était une "nouvelle", dans l'édition du recueil El jardín de senderos que se bifurcan (1941), puis dans celle de Ficciones (1944). Mais à la page suivante, un bref « Avertissement » vient nuancer la situation:

En fin de compte, Legrand n'a manifestement pas réussi à publier ces pages, si tant est qu'il s'y soit essayé. Le hasard ou quelque autre nécessité a voulu qu'elles demeurent, avec d'autres, dans un carton miraculeusement conservé au grenier d'une maison languedocienne, d'où l'on entreprend aujourd'hui de les tirer (2008: 17).

6 Cet avertissement est signé "L'éditeur, 24 décembre 2008 ", ce qui laisse apparaître que cet éditeur anonyme a retrouvé, transcrit, annoté (il y a de nombreuses «notes de l'éditeur») et donné à l'imprimerie le manuscrit abandonné par Maurice Legrand cinquante ans plus tôt. Mais la question de l'auteur ne s'arrête pas là, il convient d'ajouter le Post-scriptum dont nous parlions plus haut, qui donne à l'ensemble textuel le statut de « roman », dont il éclaire la gestation dans son rapport avec la nouvelle de Borges, et qui est signé «M. L. », initiales qui, conventionnellement, renvoient au nom de l'auteur du roman, Michel Lafon, mais qui, curieusement, sont les mêmes que celles de Maurice Legrand. Nous avons donc affaire à une triple figure d'auteur, dont la production a pour fonction de mettre en lumière la vie et l'œuvre d'un auteur inventé par Borges.

7 L'affaire est complexe, mais nous pouvons encore évoquer quelques ingrédients complémentaires. «Pierre Ménard, auteur du Quichotte » est la première nouvelle écrite 
par Borges dans des circonstances très dramatiques ${ }^{1}$ : après une septicémie qui avait failli l'emporter, Borges a très peur d'avoir perdu ses facultés créatrices et, pour se mettre à l'épreuve, il décide d'écrire dans un genre qu'il n'avait jamais pratiqué jusqu'alors, et qu'il appellera par la suite fiction. On y trouve des éléments fondateurs de l'écriture borgésienne: la problématique de l'auteur, de la création littéraire, de la mémoire, la présence du Quichotte comme un des noyaux générateurs de la littérature, la ville de Nîmes où Borges séjourna durant son adolescence, qui est un de ses lieux de prédilection en France, et tant d'autres choses que l'on trouve dans l'abondante critique suscitée par cette nouvelle.

Que Michel Lafon ait choisi Pierre Ménard comme axe structurant de son premier roman n'est ni fortuit ni insignifiant, non seulement à cause des problématiques qu'il incarne, mais aussi en tant que personnage languedocien : il l'a tiré de Nîmes vers Montpellier, la ville où lui-même était né, et en particulier vers le Jardin des plantes, dont il fait le centre de rencontres d'un cénacle d'artistes, célèbres ou inconnus, tous inspirés par cet écrivain dont l'œuvre «invisible» est bien plus importante que son œuvre «visible». Qu'il ait donné à son roman deux « auteurs » - que je préfère appeler, pour ma part, un double sujet producteur -, avant d'en ajouter un troisième dans le Post-scriptum, n'étonnera pas les lecteurs au fait de l'intérêt porté par Michel Lafon à l'écriture à quatre mains, comme le démontre l'ouvrage qu'il a écrit avec Benoît Peeters: Nous est un autre. Enquête sur les duos d'écrivains. En fait, il serait plus juste de dire que Une vie de Pierre Ménard a été écrit par Jorge Luis Borges et Michel Lafon, et qu'il réalise le rêve de Borges, toujours dénié, d'écrire un roman, et celui de Lafon, toujours poursuivi, de s'identifier à Borges.

Revenons brièvement sur les dates mentionnées au début et à la fin du roman. La première correspond à la fin de la rédaction des pages consacrées par Maurice Legrand à la vie et l'œuvre de Pierre Ménard: 24 août 1957. Dans la fiction, c'est le vingtième anniversaire du décès de l'écrivain, il se trouve que le 24 août est aussi la date de naissance de Jorge Luis Borges. La deuxième est celle de l'avertissement de l'éditeur, 24 décembre 2008 : on remarquera une certaine symétrie avec la première, et cette fois il s'agit de la date conventionnelle de la naissance de Jésus. Mais surtout, si on la met en regard de la date d'achevé d'imprimer (qui ne fait pas partie intégrante du corpus textuel), 22 octobre 2008, on observe un dysfonctionnement chronologique délibéré, puisque le texte de l'éditeur n'a pu être écrit postérieurement à l'impression du roman. Petite "frivolité » très borgésienne, comme un dernier hommage secret rendu par l'auteur multiple du roman.

\section{Structure de Une vie de Pierre Ménard}

10 À première lecture le roman laisse apparaitre une grande complexité due à son extrême fragmentation et au caractère hétéroclite des matériaux textuels qui le composent: « livre bâti un peu à la diable, ce dont j'espère que l'on ne me tiendra pas rigueur " prévient Maurice Legrand dans son avant-propos. Ces deux caractéristiques sont bien évidemment présentes dans toute l'œuvre de Borges, et il est parfaitement légitime que Michel Lafon les ait reprises dans son roman, d'autant qu'elles appartiennent aussi à l'écriture de Pierre Ménard, selon son disciple Maurice Legrand. On voit se construire une filiation de l'écriture que l'on peut décrire de la sorte: Borges invente en 1939 un personnage d'écrivain polygraphe, symboliste nîmois du début $\mathrm{du} \mathrm{xx}^{\mathrm{e}}$ siècle, auquel il attribue une œuvre visible (sans grand intérêt), et une œuvre invisible qui consiste à 
réécrire le Quichotte, non pas en le recopiant, mais en l'inventant à l'identique trois cents ans après la publication du chef-d'œuvre de Cervantes. Soixante dix ans plus tard, Michel Lafon écrit un premier roman où il se réapproprie ce personnage, lui attribue une existence réelle, en reprenant des éléments de la nouvelle et en ajoutant des éléments de son cru. Il introduit deux chaînons intermédiaires : un disciple de Ménard qui a écrit, en 1957, un livre de témoignage sur son maître, mais ne l'a pas publié; un autre personnage, "l'éditeur", dont on ignore la relation avec le précédent, trouve, cinquante ans plus tard, le manuscrit de Maurice Legrand qu'il transcrit, annote et donne à la publication en 2008. Dans la fiction, la filiation de l'écriture est différente : Cervantes, réécrit par Pierre Ménard, réécrit par Borges et par Maurice Legrand, reproduit par l'éditeur, et Michel Lafon qui se réapproprie l'ensemble de la chaîne sous forme de roman.

11 Afin d'avoir une vue d'ensemble du roman, il est nécessaire d'en proposer une cartographie avant de revenir sur certains éléments. On conserve les différentes graphies.

- Dédicace : À la mémoire de Françoise et Pedro, lecteurs acharnés. (p. 7)

- Épigraphe : citation de JORGE LUIS BORGES «Pierre Ménard, auteur du Quichotte », Fictions. (p. 9)

- AVANT-PROPOS, signé : Maurice Legrand, Montpellier, 24 août 1957. (p. 11-16)

- AVERTISSEMENT, signé : L'éditeur, 24 décembre 2008. (p 17-18)

- De la p. 19 à la p. 95 : texte de Maurice Legrand, écrit en fragments de dimensions variées séparés par un astérisque, incluant des textes d'autres auteurs, des fragments de lettres, de poèmes. Certains fragments ont des titres: Ménard traducteur; Disciples; Ménard photographique. Il y a quelques notes de bas de page, les unes signalées comme (Note de Legrand), les autres comme (Note de l'éditeur).

- De la p. 96 à la p. 116 : Pierre Ménard, le Jardin des Plantes de Montpellier (FRAGMENTS ET NOTES PRÉPARATOIRES)», dont une note de Legrand précise qu'il s'agit de "Pages perdues (abandonnées ?) par Ménard dans la maison de Castelnau-le-Lez et retrouvées par moi [... ]» (p. 96). Ce texte est écrit de la même façon que le précédent, il y a trois notes de Legrand, et une note de Ménard. Le texte se termine par une indication: (Fin des fragments du Jardin des Plantes de Montpellier).

- De la p. 117 à la p. 134 : (SUITE DES NOTES DE MAURICE LEGRAND), texte écrit de la même façon que le précédent, il y a trois notes de Legrand, et une note de l'éditeur.

- De la p. 135 à la p. 143 : Mémoire pour servir à l'histoire du Congrès, dont une note de l'éditeur précise que "Legrand joint ces notes [...] à sa "biographie", sans en révéler l'origine ni l'auteur ». Ce texte n'est pas fragmenté. Outre la première note de l'éditeur, il y a deux Notes de l'auteur du Mémoire pour servir à l'histoire du Congrès, dont la seconde est accompagnée d'une Note de Legrand. Le texte se termine par une indication : (Fin du Mémoire pour servir à l'histoire du Congrès).

- De la p. 144 à la p. 147 : (SUITE DES NOTES DE MAURICE LEGRAND), texte écrit de la même façon que celui des p. 117-134, mais sans notes.

- De la p. 148 à la p. 154 : Jorge Luis Borges, souvenirs d'avril 1919, dont une note de Legrand précise que «Borges rédige ces pages directement en français [...]. Il me les adresse au début 
des années 1950 ». Ce texte n'est pas fragmenté, il ne comporte aucune note. Il se termine par une indication : (Fin des pages de J. L. B.).

- De la p. 155 à la p. 179 : (SUITE ET FIN DES NOTES DE MAURICE LEGRAND), texte écrit de la même façon que celui des p. 144-147, avec deux notes de Legrand et deux notes de l'éditeur.

- De la p. 181 à la p. 183 : Post-scriptum, texte signé de deux initiales : M. L. On notera qu'elles peuvent renvoyer aussi bien à Maurice Legrand qu'à Michel Lafon. Une note, non signée, pointe la mise en parallèle de « ce roman » avec la nouvelle de Borges citée en épigraphe.

- Par ailleurs, on signalera que l'achevé d'imprimé, en dernière page, porte la date du 22 octobre 2008, à mettre en regard de la date de l'Avertissement signé par l'éditeur, le 24 décembre 2008 (p. 18).

L'ensemble textuel dont on vient de dresser la cartographie est désigné comme «roman », non seulement par le péri-texte de la maison d'édition, mais aussi, de façon insistante, par M. L. dans son Post-scriptum qui donne des indications précieuses sur l'écriture de ce texte, ses multiples sources, sur les relations intimes de l'auteur avec la ville de Montpellier et ses alentours, avec le Jardin des plantes et ses histoires prodigieuses. Il y proclame le caractère fictif de l'ensemble, en terminant sur ces mots : "Tout, dans une fiction, absolument tout, comme on le voit, est bel et bien fictif»(p.183), alors qu'il a par ailleurs donné de nombreux éléments et sources « historiques ». Jeu très borgésien, qui en outre s'autorise d'un entretien de l'auteur avec Jorge Luis Borges :

Lors du long entretien qu'il m'accorda au printemps 1983 à Paris, à l'Hôtel de la rue des Beaux-Arts, Borges me confirma qu'il connaissait ma ville natale et qu'il y avait fait étape à cette époque-là. Je n'avais de toute façon nul besoin de cette savoureuse confirmation pour inventer la suite: les Jardiniers et les Templiers, le Congrès souterrain (et le Mémoire pour servir à son histoire), la réalité de Pierre Ménard, sa fréquentation assidue du Jardin des Plantes de Montpellier, son influence sur Borges et sur tant d'autres, les «souvenirs d'avril 1919 », les conversations et les confidences, les correspondances, les paperoles, les journaux... » (2008 : 182)

Il est clair que ce qui est en jeu ici c'est l'inextricable imbrication entre réalité et fiction, mais aussi entre théorie et fiction. Michel Lafon a construit un artefact littéraire qu'il appelle roman (" une facilité - et presque une faiblesse»), mais qui reste fidèle à la théorie et à la pratique d'écriture de son maître Borges. À la fin, «en écrivant les dernières lignes de ce post-scriptum », il se rend compte qu'il est un double de Ménard et qu'il s'est mis en situation de réécrire la nouvelle de Borges à la façon dont Pierre Ménard a réécrit quelques fragments du Quichotte. Bien sûr, le lecteur de Une vie de Pierre Ménard s'en était rendu compte avant de lire le post-scriptum.

Fragmentation, doubles et dédoublements en cascade, hybridations génériques multiples, brouillage des frontières, filiations intellectuelles et artistiques, attributions douteuses, influences et plagiats assumés ou déniés, chronologies fantaisistes : tout y est. Toutes ces caractéristiques de l'œuvre de Borges se retrouvent dans le roman de Lafon : c'est à la fois un fervent hommage et un tour de prestidigitation intellectuel. Il faudrait relever minutieusement les innombrables indices qui balisent le roman pour en faire un palimpseste et un texte à clés multiples, car Borges n'est pas la seule clé, et on doit se demander pourquoi, par delà l'invention géniale de ce qu'est, fondamentalement, l'écriture, cette nouvelle s'est constituée en noyau générateur de Une vie de Pierre Ménard.

De toute évidence, c'est le personnage de l'intellectuel nîmois qui a fasciné Michel Lafon au point de le contraindre à revendiquer pour lui le statut de personne réelle, que lui avait conféré puis dénié l'écrivain argentin. Comme nous l'avons déjà observé, le Nîmois 
devient Montpelliérain dans le roman, non seulement parce qu'il finit par s'installer dans la capitale languedocienne, mais surtout à cause de sa passion pour le fameux Jardin des plantes, dont il fait une réplique du Jardin d'Akadémos. Michel Lafon, Montpelliérain de naissance et de cœur, ne pouvait que s'identifier à ce poète, critique, érudit, épistolier et maître à penser modeste et discret qui conçoit le projet pharamineux de réinventer le Quichotte après l'avoir méthodiquement oublié. Il campe auprès de Ménard un disciple montpelliérain, plein d'admiration et de sollicitude, qui l'accompagne jusqu'à sa mort et, vingt ans plus tard, rédige le manuscrit fragmentaire d'une biographie du maitre, destinée à le restaurer dans son existence historique. D'emblée on voit l'importance du rapport maître-disciple qui fonde l'écriture, ainsi que les relations amicales et intellectuelles que Ménard entretient avec André Gide, Paul Valéry et d'autres écrivains qui partagent ses promenades dans le Jardin des plantes. C'est là aussi qu'il fait un jour la connaissance d'un jeune écrivain argentin, très prometteur, avec qui il entretiendra des liens épistolaires pendant une vingtaine d'années. Ce rapport maître-disciple est une particularité du texte romanesque, ce qui implique qu'il soit structurant dans la lectureinterprétation-création que Lafon réalise à partir de la nouvelle, c'est-à-dire qu'il soit important dans sa conception de l'écriture et de la transmission littéraire.

La relation de Michel Lafon à l'œuvre et à la personne de Borges est bien un rapport maî tre-disciple: en effet, il ne s'agit pas seulement d'une admiration littéraire pour ces nouvelles lues depuis l'adolescence et tout au long de sa vie, mais aussi d'une adhésion intellectuelle à la théorie de la littérature qui sous-tend l'ensemble de l'œuvre, et d'un intérêt affectif pour une existence entièrement consacrée à l'écriture, ou mieux, au lirécrire, à l'échange intellectuel et amical avec quelques personnes choisies comme Silvina Ocampo (à qui est dédiée la nouvelle qui nous occupe) ou Adolfo Bioy Casares, son alter ego en écriture. Il convient de rappeler que Michel Lafon a entretenu une longue amitié avec Bioy, dont il admirait également l'œuvre et la personnalité, prenant en quelque sorte le relais de Borges après 1986. La pratique de l'écriture «à quatre mains » de Borges et Bioy, devenue un véritable mythe littéraire, a aussi fasciné Lafon qui a signé avec Benoît Peeters Nous est un autre. Enquête sur les duos d'écrivains. La présence de Bioy dans le roman est d'ailleurs soulignée dans le post-scriptum: "L'île de Bloy est un clin d'œil à Edgar Allan Poe et à Adolfo Bioy Casares - sa première phrase est la transposition en français du début de L'Invention de Morel. (Je confirme ici le projet de publier un jour ce roman entrevu.) » (181) Il apparaît clairement que Bioy est aussi un maître pour Lafon, et que le projet d'écrire L'île de Bloy, qu'il attribue à Ménard mais qu'il revendique pour lui, est un double du projet mené à son terme dans Une vie de Pierre Ménard. Le destin a voulu que, à l'instar de Maurice Legrand, le disciple de Ménard, qui n'a pas pu (ou pas voulu) publier la biographie du maître, Michel Lafon n'ait pas pu publier L'île de Bloy, dont le manuscrit est peut-être dans quelque tiroir de sa maison de Voreppe, ou au fond d'un carton « dans le grenier d'une maison languedocienne ».

Mais il y a un autre maitre, plus difficile à identifier parce qu'il est moins connu, mais dont je décèle la présence diffuse parce qu'il a été aussi le mien, c'est Maurice Molho (Salonique, 1922 - Paris, 1995), linguiste, traducteur et critique littéraire hors-pair, qui accompagna la carrière de Michel depuis l'École normale de Saint-Cloud jusqu'à la thèse d'État. Non seulement il a prêté son prénom à Legrand, mais encore a-t-il largement inspiré la théorie de la traduction développée par Ménard dans le fragment Ménard traducteur (p. 61-64): 
Il a sa méthode, que l'un de ses amis, un spectaculaire professeur en Sorbonne, a baptisée ménardisation. Ménard a opté, une fois pour toutes, pour une absolue fidélité à l'original. Il ne condamne pas seulement, comme tout un chacun, les «belles infidèles». Il réprouve même les traducteurs qui, globalement fidèles, s'écartent en quelques points de l'original, au nom du "bon français ", de "ce qui se dit» (et surtout de «ce qui ne se dit pas »), des habitudes, des normes et autres bonnes mœurs littéraires. Il veut, quant à lui, que la phrase française suive à tout moment la phrase espagnole d'origine, jusque dans la disposition des mots, la longueur et le couleur des vocables, la musique, le rythme, la respiration... (2008 : 61-62)

18 Ce discret hommage au maître Molho, qui est identifié au maître Ménard, est d'autant mieux venu que Maurice était aussi un grand spécialiste de Cervantes, sur qui il a écrit des études remarquables, et que c'est précisément un texte de Cervantes que Ménard veut réinventer. Où l'on voit que la fiction et la réalité historique se complètent avantageusement. Puisque le hasard n'existe pas, on peut très bien penser que Borges, en écrivant "Pierre Ménard, auteur du Quichotte», a inventé Maurice Molho écrivant sur la première phrase du Quichotte cinquante ans plus tard (1989 : 83-92).

19 L'un des chapitres du roman attire particulièrement l'attention du lecteur dans la mesure où il se donne comme des pages rédigées par Jorge Luis Borges, directement en français, sur le souvenir de sa rencontre avec Pierre Ménard dans le Jardin des plantes de Montpellier, un jour d'avril 1919. La famille de Borges fait une étape à Montpellier sur le chemin de Genève, où ils sont restés pendant la durée de la guerre, et l'Espagne, d'où ils doivent s'embarquer pour Buenos Aires. On se souvient que Lafon précise, dans le Postscriptum, que Borges, dans un entretien de 1983, lui confirma qu'il était passé dans la capitale languedocienne à cette époque. Souvenirs d'avril 1919 est accompagné d'une longue note de Maurice Legrand où il explique les circonstances dans lesquelles il a reçu ces pages de Borges, « au début des années cinquante », et où il développe une théorie de l'influence et de la filiation littéraires très intéressante :

Quant à la proximité de leur style avec tant de pages de ce dossier, bref avec le style de Ménard (je n'ose parler du mien), elle peut laisser perplexe. Qui influence qui à la fin? Comment résister à la tentation égale de voir Borges en inspirateur de Ménard, et Ménard en précurseur de Borges? Pour moi mon siège est fait : nous sommes tous des enfants de Ménard, des réécrivains, inaboutis mais fidèles et fascinés, de ce Ménard qui lui-même s'était choisi le parrainage de Fabre, qui pour sa part réécrivait Virgile. Tous élèves d'Aristote, de Pythagore ou peut-être, qui sait, de Zénon - «Zénon! Cruel Zénon! Zénon d'élée!»-, inépuisable et fabuleux Zénon... (2008: 149)

20 Tout est dit: Ménard, Borges, Legrand, Lafon se réécrivent l'un l'autre, dans n'importe quel ordre, et réécrivent tous les grands maîtres jusqu'à Zénon d'Élée, invoqué par le célèbre vers du "Cimetière marin » de Paul Valéry, un autre promeneur du Jardin des plantes. Toute écriture est réécriture, tout livre est un palimpseste. Le Jardin des plantes de Montpellier est aussi une métaphore de la réécriture - ou du lirécrire -, comme le souligne un autre bref chapitre du roman, Mémoire pour servir à l'histoire du Congrès.

21 Une vie de Pierre Ménard est non seulement une réécriture de la nouvelle « Pierre Ménard, auteur du Quichotte ", mais aussi de Borges ou la réécriture, car on écrit toujours le même texte, selon des modalités différentes. Michel Lafon avait choisi très tôt de réécrire Jorge Luis Borges et de vivre dans un Jardin languedocien peuplé de poètes, d'érudits, de penseurs élégants et amènes. C'est ainsi qu'il restera dans notre souvenir. 


\section{BIBLIOGRAPHIE}

BORGES Jorge Luis, Euvres complètes, édition de Jean-Pierre Bernès (1993), Paris : NRF Gallimard.

LAFON Michel (1990), Borges ou la réécriture, Paris : Seuil Poétique.

LAFON Michel \& Peeters Benoît (2006), Nous est un autre. Enquête sur les duos d'écrivains, Paris :

Flammarion.

LAFON Michel (2008), Une vie de Pierre Ménard, Paris : NRF Gallimard.

MolHo Maurice (1989), «Utopie et uchronie : sur la première phrase du Don Quichotte », Le temps du récit, Madrid : Annexe aux Mélanges de la Casa de Velázquez, Rencontres 3.

\section{NOTES}

1. Voir la note très circonstanciée dans J. L. Borges, CEuvres complètes (1993 : 1570).

\section{RÉSUMÉS}

Une vie de Pierre Ménard (2008) est le premier roman de Michel Lafon, le sort a voulu que ce fût aussi le dernier. Son dessein est de rendre au personnage inventé par Jorge Luis Borges dans sa nouvelle «Pierre Ménard, auteur du Quichotte» (1939) la réalité historique qu'il lui a d'abord donnée, puis déniée. Le roman se construit autour d'un savant mélange entre les éléments empruntés à la nouvelle, et ceux que Lafon a inventés, avec une structure fragmentée et apparemment chaotique. Ce sont ces caractéristiques que l'on essaye de décrire et d'analyser dans cette œuvre que l'on peut considérer comme le testament littéraire de Michel Lafon.

Une vie de Pierre Ménard (2008) is Michel Lafon's first novel, if it unfortunately so happened that it was also his last. His aim was to reinvest the character invented by Borges in his short story "Pierre Ménard, author of the Quixote" (1939) with a historical presence which he had given him in the first place and subsequently denied. The work is built on the subtle mixture of elements from Borges' story, with others due to Lafon's invention, with a kaleidoscopic and chaotic structure: those are the features described and analyzed in this work, which can be taken as Lafon's literary testament.

Une vie de Pierre Ménard (2008) es la primera novela de Michel Lafon, el destino ha decidido que fuese también la última. Se trata de devolverle al personaje inventado por Jorge Luis Borges en su cuento «Pierre Menard, autor del Quijote» (1939) la realidad histórica que éste le dio y luego le negó. La novela se construye en torno a una compleja hibridación entre los elementos tomados del cuento, y los que Lafon inventó, con una estructura fragmentada y aparentemente caótica. 
Son estas características las que se trata de describir y de analizar en esta obra que se puede considerar como el testamento literario de Michel Lafon.

INDEX

Palabras claves : Jorge Luis Borges, Pierre Menard - autor del «Quijote», reescritura, fragmentación, testamento literario

Mots-clés : Jorge Luis Borges, Pierre Ménard - auteur du « Quichotte », réécriture, fragmentation, testament littéraire

Keywords : Jorge Luis Borges, Pierre Ménard - author of the "Quixote", kaleidoscopic structure, literary testament

\section{AUTEUR}

\section{MILAGROS EZQUERRO}

Université Paris-Sorbonne - Paris 4 